\title{
Long QT syndrome mimicking refractory epilepsy: case report and literature review
}

\author{
Huicong Kang ${ }^{1}$, Li-li Lan ${ }^{1}$, Yu-chao $\mathrm{Jia}^{1}$, Cun $\mathrm{Li}^{1}$, Yong-kang Fang ${ }^{1}$, Sui-qiang Zhu ${ }^{1}$, and \\ Heidi Kirsch ${ }^{2}$ \\ ${ }^{1}$ Tongji Hospital of Tongji Medical College of Huazhong University of Science and \\ Technology \\ ${ }^{2} \mathrm{UCSF}$
}

August 17, 2020

\begin{abstract}
Epileptic seizures can be difficult to distinguish from other etiologies that cause cerebral hypoxia, especially cardiac diseases. Long QT syndrome (LQTS), especially LQTS type 2 (LQT2), frequently masquerades as seizures because of the transient hypoxia caused by ventricular arrhythmia. Early and correct diagnosis of LQTS effectively prevents inappropriate treatment with anti-epileptic drugs (AEDs) and sudden death. We report a case of congenital LQT2 with potassium voltage-gated channel subfamily $\mathrm{H}$ member 2 gene (KCNH2) mutation misdiagnosed as refractory epilepsy and treated with various AEDs for 22 years. The possibility of cardiac arrhythmia was suspected after the electrocardiograph (ECG) monitor in emergency room showed paroxysmal ventricular tachycardia during attacks. Atypical seizure like attacks with prodromal uncomfortable chest sensation and palpitation, triggered by auditory stimulation, and typical ventricular tachycardia monitored by ECG raised suspicion for LQT2, which was confirmed by exome sequencing. Although the patient rejected implantation of an implantable cardioverter defibrillator, $\beta$-blocker was given and the syncope only attacked 1-2 per year when there was an incentive. Our case illustrates how long LQTS can masquerade convincingly as epilepsy and be treated wrongly with AEDs, putting the patient at high risk of sudden death. Careful ECG evaluation is recommend for both patients with first seizure and those with refractory epilepsy.
\end{abstract}

\section{Hosted file}

Kang-manuscript.doc available at https://authorea.com/users/346919/articles/476085-long-qtsyndrome-mimicking-refractory-epilepsy-case-report-and-literature-review 

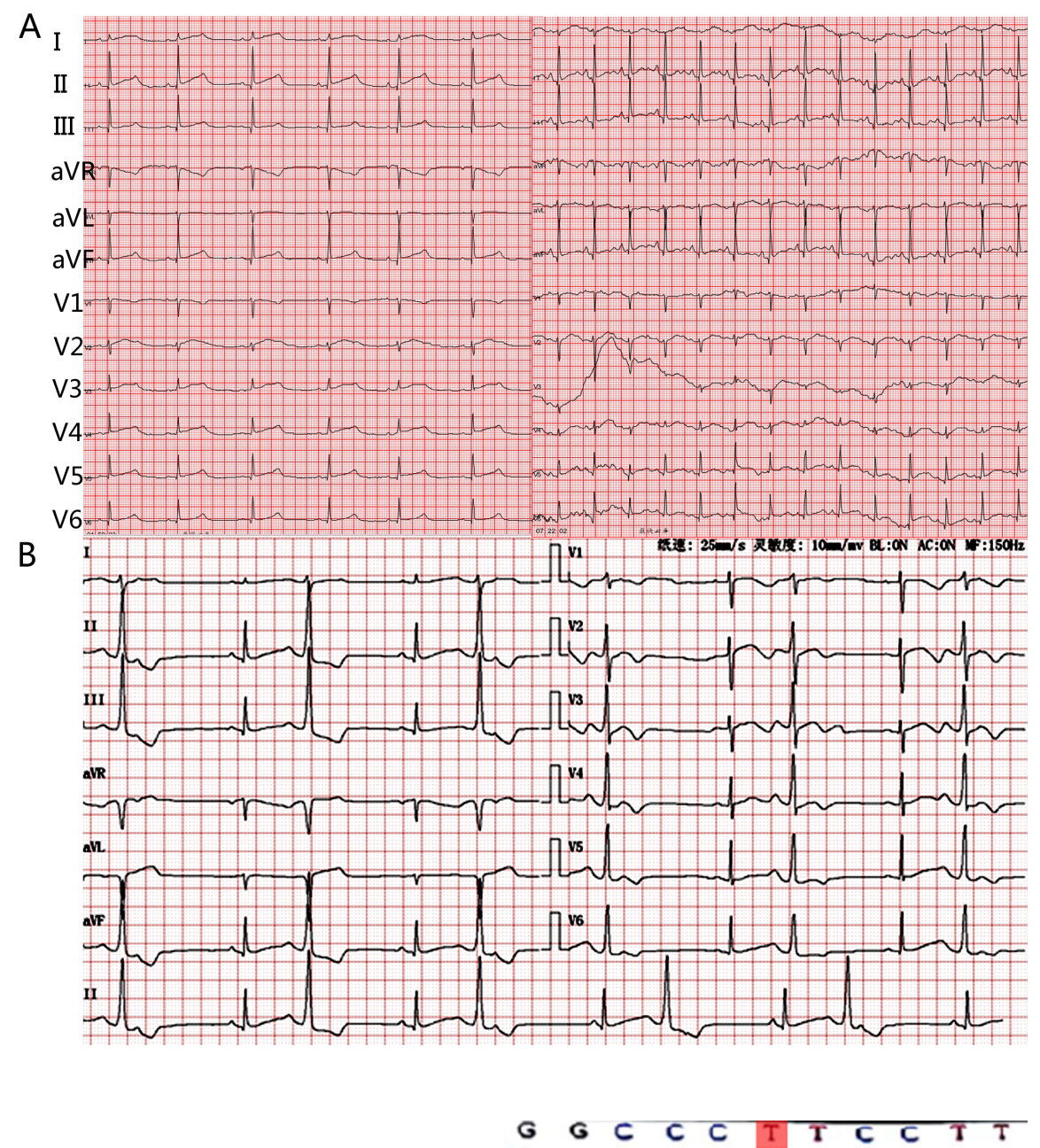

Patient and her mother (mutation)

Patient' $s$ father (wildtype)

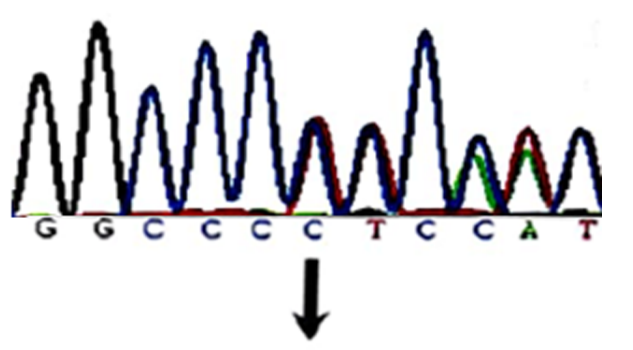

$$
\text { NWMOMW }
$$

2 